\title{
Hydra as a Model Organism
}

MANY phenomena occurring during the development of multicellular organisms imply intercellular communication. For example the ability to regulate-to produce a standard form independent of size-implies that cells must know their relative position within an embryo. If the two blastomeres of a frog embryo are separated after its first division each will regulate to produce a normal, but small, embryo. Many theories for the mechanism of signalling in embryos have been produced; the oldest, and simplest, is that there is a concentration gradient of a "morphogen" from the animal to vegetal poles, and that cells can measure their ambient concentration and then behave according to their position in the gradient. The production of a stable gradient implies that there is a source of the morphogen at one pole of the embryo and a sink at the other. This kind of model has been used, for example, to explain the production of patterns in the insect integument.

A radically different class of model uses a rapidly propagated signal to supply information to embryonic cells. Whereas such a system might have many advantages, the only example of its use in a real organism is in the signal controlling aggregation in some cellular slime moulds. It is questionable whether one can safely draw analogies between the development of slime mould and that of more complex organisms. On the other hand, it is clearly important to understand the processes controlling development in simple organisms, or in circumscribed situations in metazoan embryos. For this reason many simple "model" systems are popular amongst developmental biologists. Two articles in a recent issue of Nature New Biology are concerned with hydra, which already has a distinguished career as a model organism.

Hydra can regenerate and regulate. If the head of a hydra is removed a new head regenerates at the anterior cut surface. This regeneration does not require cell division, but stems from a reorganization of the tissues of the hydra. Further, the presence of an extra head nearby can inhibit head regeneration at a cut surface. The implication is that a head acts as a source of some substance that actively inhibits head formation, and possibly also supplies "positional information" to cells within the hydra. When a head is removed the gradient of inhibitor relaxes and, it is postulated, when the concentration of inhibitor has fallen below a threshold value, which is a function of axial position, new head formation is initiated. If these postulates, due to Wolpert and his colleagues, are correct, then hydra does indeed provide an ideal experimental system for investigating signalling during the control of a developmental process.

Wolpert, Clarke and Hornbruch (Nature New Biology, 239,$101 ; 1972$ ) have investigated the rate of propagation of the inhibitory signal. Their basic experiment involves the determination of how long an extra head must be grafted into the axis of a hydra in order to prevent head regeneration when the hydra's original head is removed. In this way one can measure the time required for a head to set up its own inhibitory field. By varying the position on the axis at which the extra head is grafted it is also possible to find the time required for inhibition as a function of distance. At first sight it would seem that this time should increase as the square of the axial distance if inhibition depends on the diffusion of a substance from a head region. A grafted head is, however, supplying inhibitor to a field which already contains an original inhibitor gradient, so the relationship is more complicated.

The results of Wolpert et al. show that the times for signal transmission get quite long, and indeed are not consistent with a rapid signal such as an electrical or chemical impulse propagated from cell to cell. They have made reasonable assumptions about the shape and production of the underlying inhibitor gradient and have used these in a computer simulation of the signalling process. The simulation shows, quite successfully, that diffusion of a relatively small molecule, such as a nucleotide, could well account for the propagation of the inhibitory signal.

This is an important result, although one should be careful to avoid reading too much into it. Such simulation involves enough free parameters to make it difficult to do more than show a consistency between the model and the experimental data. In this case, for example, it would be necessary to determine the chemical nature of the inhibitory morphogen before one could make unequivocal tests of the model. This, of course, is a problem besetting all theoretical interpretations of biological events; biological systems are so complex that it is possible to pick enough variables to fit any model to the facts.

A further capability of hydra is reconstitution of an individual from dissociated cells, as Gierer et al. show (Nature New Biology, 239, 98; 1972). Again, this provides an important model system for examining the development of polarity and positional information, and the subsequent control of cell movement and differentiation. In their experiments, Gierer et al. find that dissociation of hydra, by mechanical disruption, into single cells and small groups of cells can be followed by reaggregation and regeneration. They show that the composition of groups of cells or cellular constituents determines polarity within the regenerating organism, defining its axis. Polarity does not depend on the orientation of single cells. Again, a mechanism for setting up positional information to control development is probably involved. Furthermore, the earliest stages of reorganization do not require cell division, although a minimal proportion of epithelial cells is needed. Cell division does appear to be necessary for some subsequent differentiation. The system is therefore useful for investigating the control of differentiation as well as for determining how cells know and remember their position within a hydra axis. As Gierer et al. show that even dissociated cells show some memory of their original position, it should be possible to find what kinds of biochemical treatment before reaggregation can disrupt this memory. Presumably the relevant biochemical mechanism is related to the mechanism responsible for the inhibitory signal, emanating from the head region, investigated by Wolpert.-From a Correspondent. 